\title{
Transmission of microwaves through a stepped subwavelength slit
}

\author{
Matthew J. Lockyear, ${ }^{\text {a) }}$ Alastair P. Hibbins, and J. Roy Sambles \\ Electromagnetic Materials Group, School of Physics, University of Exeter, Exeter EX4 4QL, \\ United Kingdom
}

(Received 11 November 2007; accepted 23 November 2007; published online 18 December 2007)

The transmission of normally incident plane wave microwaves through a single stepped subwavelength slit in a thick metal plate is explored. The presence of the step substantially increases the radiation wavelength which may be resonantly transmitted to well beyond twice the plate thickness. Insight into the resonant behavior is provided through analysis of field solutions produced by a finite element model. (C) 2007 American Institute of Physics. [DOI: 10.1063/1.2824458]

In recent years there has been much interest in the "enhanced" transmission phenomena of subwavelength apertures in metals, structured or otherwise. ${ }^{1-15}$ Unlike subwavelength holes, which rely on either diffraction or surface wave excitation to enhance the evanescent tunnelling process through the holes, slits support a propagating transverse electromagnetic (TEM) waveguide mode without cut off. ${ }^{16}$ This mode gives strong transmission through such structures at well defined wavelengths. ${ }^{8}$

For a single subwavelength slit in a thick metal substrate, weak coupling to the TEM occurs at the slit aperture due to diffraction of the incident field. Radiation, once within the slit, does not couple efficiently back out into free space due to a mode-shape mismatch, resulting in the open ends of the slit acting as highly reflecting mirrors that can support a series of standing waves. It is these Fabry-Perot (FP)-like modes that provide strong resonant enhancement at selective wavelengths to an otherwise weak transmission channel. In the limit of infinitesimal slit width and perfect conductors, peaks in the transmission will be found according to $\lambda_{N}$ $=2 n l / N$, where $\lambda_{N}$ is the wavelength of the $N$ th order mode, $n$ is the refractive index within the slit, and $l$ is the thickness of the metal plates (separation between the two partially reflecting planes). (Real structures exhibit a generally small reduction in this predicted frequency ${ }^{9,17}$ through two effects, one an end effect due to the finite width of the slit, the other due to wave front curvature within the slit through the finite conductance of the metal.) In the ideal case through quantization of the mode along the slit length, there is an upper limit of approximately twice the sample thickness (times the refractive index of the material filling the slit) for the longest wavelength transmission resonance. Here, we show that the addition of a suitable step structure within the slit results in the resonant wavelength of the fundamental mode being dramatically increased.

Three experimental samples studied are based upon an air-filled slit formed from two aluminium plates of dimensions $400(x) \times 300(y) \times 10(z) \mathrm{mm}^{3}$. When arranged with $y z$ faces aligned and separated by incompressible polyester spacers in the $z$ direction, a slit of length $l=10 \mathrm{~mm}$, breadth $b=300 \mathrm{~mm}$, and slit width $w$ is formed in an otherwise opaque metal screen. The reference sample (A, Fig. 1) consists of a subwavelength slit with no additional structuring. Sample (B), (Fig. 2) is identical to sample (A) with the ex-

${ }^{a)}$ Electronic mail: m.j.lockyear@exeter.ac.uk. ception of a $3 \mathrm{~mm}$ wide, $0.36 \mathrm{~mm}$ deep step milled into the yz face of one of the substrates that extends throughout the entire breadth of the sample $(300 \mathrm{~mm})$. Sample (C) has a notch milled into both surfaces (Fig. 3). Incident radiation is linearly polarized with its electric field vector orientated in the $x$ direction (normal to the slit). Transmission is recorded as a function of frequency for different slit widths for all three samples.

The upper inset of Fig. 1 shows the relative transmission (circles) of sample (A) as a function of frequency and for a range of slit widths between 75 and $600 \mu \mathrm{m}$ in $25 \mu \mathrm{m}$ increments. Note the strong resonant features which compare in position and form very well with the predictions from a finite element method (FEM) modeling code $^{18}$ (solid lines). The calculated fields at the resonant frequencies [Fig. 4(a)] show these transmission maxima all to be the fundamental FP mode. We note the narrowing of the resonance and the reduced transmission with decreasing slit width. However, of more interest in this study is the frequency of the fundamental resonance (Fig. 1). It is clear that for this slit, without structure, the resonant frequency decreases monotonically with increasing slit width due to an aperture effect. ${ }^{9}$

The resonant frequency of the fundamental mode of sample (B) (circles) as a function of slit width is displayed in Fig. 2 together with the modeled response. On comparison of Fig. 1 with Fig. 2 it is clear that the addition of the step to one of the metallic substrates dramatically changes the resonant behavior observed. Rather than reducing monotonically with increasing slit width, the fundamental resonance sup-

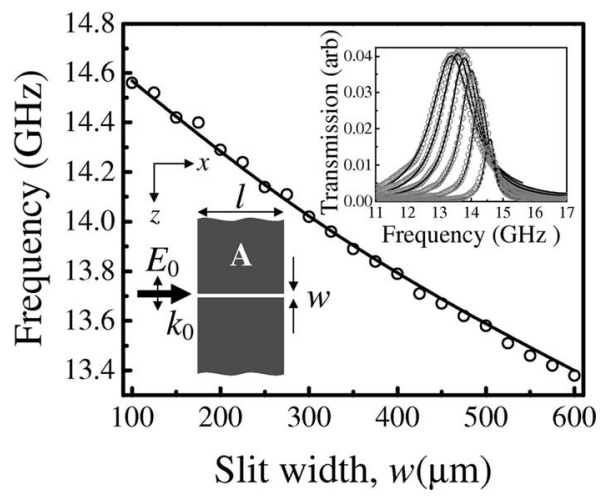

FIG. 1. Resonant frequency as a function of slit width $w$ for the fundamental resonance of sample (A) (lower inset). The experimental data (circles) and predictions of a FEM model (solid line) are shown. Upper inset: The modeled and experimental transmission spectra for the fundamental resonance. 


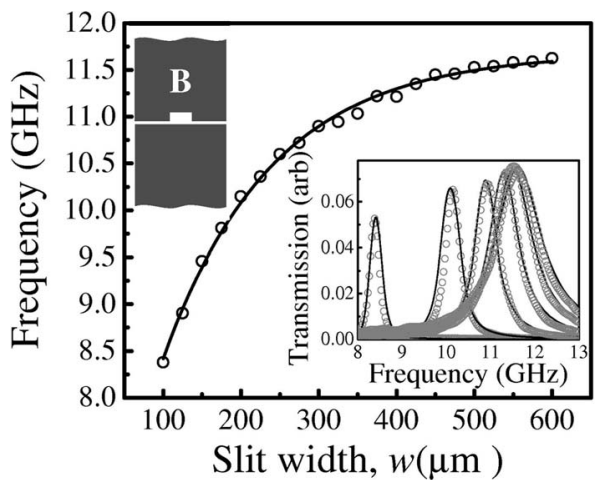

FIG. 2. Resonant frequency as a function of slit width $w$ for the fundamental resonance of sample (B) (upper inset). The experimental data (circles) and the predictions of a FEM model are shown (solid line). Lower inset: The modeled and experimental transmission spectra for the fundamental resonance.

ported by sample (B) increases in frequency. Indeed, the resonant frequency of the fundamental mode supported by sample (B) is always lower than that of sample (A), for any given slit width. For example, the fundamental resonance of sample (A) occurs at $14.6 \mathrm{GHz}$ when $w=100 \mu \mathrm{m}$, whereas the same slit width for sample (B) gives the resonance at $8.4 \mathrm{GHz}$. This striking reduction is enhanced further with sample (C), which has an identical step in the other metallic substrate (Fig. 3). Remarkably, at a slit width of $100 \mu \mathrm{m}$, the resonant frequency of the fundamental FP like mode has been reduced by $53 \%$ compared to the unstructured case. In order to understand the effect of the additional structure within the slit, it is useful to investigate the electromagnetic response of the structure on resonance using the FEM model.

Figure 4(a) shows the modeled $E_{x}$ and $H_{y}$ field calculated on a line passing midway between and parallel to the two metallic substrates of sample (A), and extending $3 \mathrm{~mm}$ beyond each end of the slit. The field strengths are represented in terms of an enhancement over the injected field strength, and calculated at a phase corresponding to maximum enhancement. Firstly, we note that the electric field strength has a maximum at the apertures of the slit, while the magnetic field strength has a maximum in the central region of the slit. This is as one would expect given "magneticwall" boundary conditions at each end of the slit, ${ }^{19,20}$ and the standing wave nature of the resonance supported (i.e., the

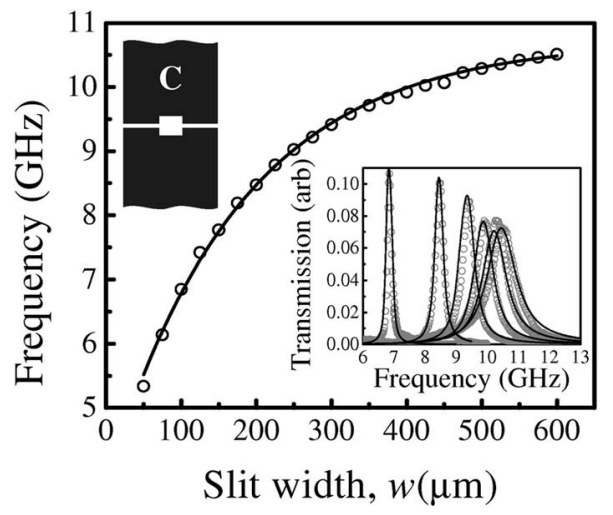

FIG. 3. Resonant frequency as a function of slit width $w$ for the fundamental resonance of sample (C) (upper inset). The experimental data (circles) and the predictions of a FEM model are shown (solid line). Lower inset: The modeled and experimental transmission spectra for the fundamental resonance of sample (C). ever, it is a similar type of "field expansion" at the steps
Downloaded 15 Apr 2008 to 144.173.6.75. Redistribution subject to AlP license or copyright; see http://apl.aip.org/apl/copyright.jsp
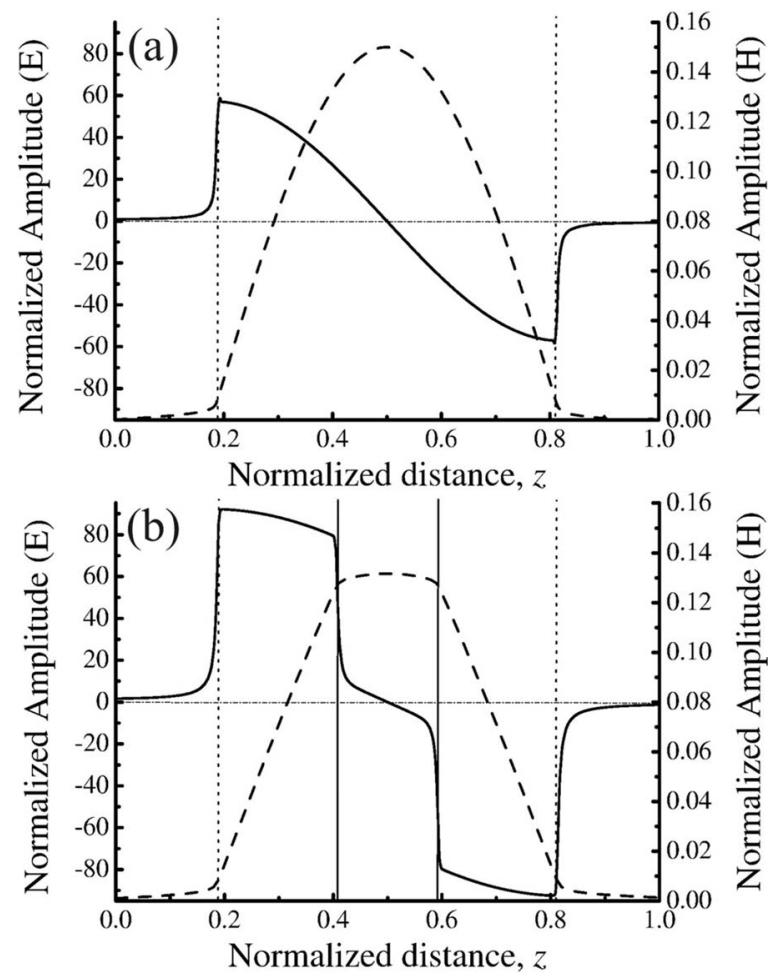

FIG. 4. Modeled enhancement of the $E_{x}$ (solid line) and $H_{y}$ (broken line) field components within the slit region of (a) sample (A) and (b) sample (C). Fields are calculated at a phase corresponding to maximum enhancement over a line passing midway between the metallic planes constituting the slit and extends $3 \mathrm{~mm}$ past the slit apertures. The vertical dotted lines are representative of the position of the apertures of the slit, whereas the solid vertical lines mark the beginning and end of the stepped cavity.

maxima in the $E$ and $H$ fields are separated by $\sim \pi / 4$ radians of phase both spatially and temporally). Further, it is in the region of the slit apertures that the greatest gradient in the $E$-field strength occurs. In this region, the incident plane wave is matched to the mode within the slit, via the strong field gradient associated with diffraction at the slit aperture. There are only two nonzero components of the incident field along the midplane of the slit $E_{x}$ and $H_{y}$. However, this is not the case at the slit apertures. In this region, the strong diffraction of the incident field into the slit gives rise to a nonzero $E_{z}$ component, and also a derivative of $E_{z}$ with respect to $y$. In this region, Maxwell's equation $\nabla \wedge \underline{E}=-\mu \partial H / \partial t$, which may be written as

$$
\left|\frac{\partial E_{z}}{\partial y}-\frac{\partial E_{y}}{\partial z}\right|=-\frac{2 \pi c \mu}{\lambda}\left|H_{x}\right|
$$

is solved with a much faster gradient of fields than is otherwise allowed in free space. This is because both $\partial E_{z} / \partial y$ and $\partial E_{y} / \partial z$ are very large but nearly cancel, varying rapidly over a distance of the order of the width of the slit (which is much smaller than the incident wavelength). The $\pi / 2$ phase change required to satisfy the matching of the cavity mode and the plane wave propagating in free space is then compressed into a distance of the order of the slit width. (A comprehensive investigation of the fields and boundary conditions associated with an array of such unstructured metallic slits by the current authors may be found in Ref. 19 together with a comparison to the more conventional etalon.) How- 
within the slit which gives rise to the effective lengthening of the stepped slits.

Figure 4(b) show the predicted $E$ - and $H$-field enhancements associated with sample (C) (steps in both substrates positioned centrally within the slit) at the resonant frequency of the fundamental mode $(6.85 \mathrm{GHz})$. On comparison of Figs. 4(a) and 4(b), we note several striking differences. Firstly, the $E$ and $H$ fields are now clearly separated spatially, with the $E$ field being strongly confined within the two narrow sections of the slit (the highly capacitive regions) and a region of nearly constant high $H$ field existing within the stepped region. Importantly, the addition of the steps results in two additional regions over which steep field gradients are obtained. These field gradients are again associated with the diffraction and expansion of the field at either end of the stepped section of the cavity. As the field expands into the wider region of the cavity, they match up with a longitudinal oscillation of surface current density existing on each metallic wall. ${ }^{19}$ At any instant, the current flow on each of the two metallic surfaces must be in opposite directions, forming an inductive current loop within the stepped region thereby increasing the effective inductance of the system. This increase in inductance results in a reduction of the resonant frequency of the mode, since, from lumped circuit theory, the notched slit may be thought of as a capacitive part $C$ (the narrow entrance and exit channels of the slit) and an inductive part $L$ (the stepped cavity) with a resonant frequency to first order given by $1 / \sqrt{L C}$.

To summarize, it is shown that with the addition of a suitable step, the fundamental resonant wavelength of a subwavelength slit may be increased by approximately $50 \%$. This shows that suitable internal structuring of subwavelength slits is a route to reducing the dimensions of electro- magnetic filters or increasing the operational bandwidth of truncated guides.

The authors acknowledge the support of the EPSRC.

${ }^{1}$ T. W. Ebbesen, H. J. Lezec, H. F. Ghaemi, T. Thio, and P. A. Wolff, Nature (London) 391, 667 (1998).

${ }^{2}$ H. F. Ghaemi, T. Thio, D. E. Grupp, T. W. Ebessen, and H. J. Lezec, Phys. Rev. B 58, 6779 (1998).

${ }^{3}$ T. Lopez-Rios, D. Mendoza, F. J. Garcia-Vidal, J. Sanchez-Dehesa, and B. Pannetier, Phys. Rev. Lett. 81, 665 (1998).

${ }^{4}$ U. Schroter and D. Heitmann, Phys. Rev. B 58, 15419 (1998).

${ }^{5}$ J. A. Porto, F. J. Garcia-Vidal, and J. B. Pendry, Phys. Rev. Lett. 83, 2845 (1999).

${ }^{6}$ P. Lalanne, J. Hugonin, S. Astilean, M. Palamaru, and K. Moller, J. Opt. A, Pure Appl. Opt. 2, 48 (2000).

${ }^{7}$ E. Popov, M. Neviere, S. Enoch, and R. Reinisch, Phys. Rev. B 62, 16100 (2000).

${ }^{8}$ H. E. Went, A. P. Hibbins, J. R. Sambles, C. R. Lawrence, and A. P. Crick, Appl. Phys. Lett. 77, 2789 (2000).

${ }^{9}$ Y. Takakura, Phys. Rev. Lett. 86, 5601 (2001).

${ }^{10}$ F. Yang and J. R. Sambles, Phys. Rev. Lett. 89, 063901 (2002).

${ }^{11}$ A. P. Hibbins, J. R. Sambles, and C. R. Lawrence, Appl. Phys. Lett. 81, 4661 (2002).

${ }^{12}$ P. Stavrinou and L. Solymar, Opt. Commun. 206, 217 (2002).

${ }^{13}$ H. J. Lezec, A. Degiron, E. Devaux, R. A. Linke, L. Martin-Moreno, F. J. Garcia-Vidal, and T. W. Ebbesen, Science 297, 820 (2002).

${ }^{14}$ L. Martin-Moreno, F. J. Garcia-Vidal, H. J. Lezec, A. Degiron, and T. W. Ebbesen, Phys. Rev. Lett. 90, 167401 (2003).

${ }^{15}$ M. J. Lockyear, A. P. Hibbins, and J. R. Sambles, Phys. Rev. Lett. 94, 193902 (2005).

${ }^{16}$ R. E. Collin, Field Theory of Guided Waves, 2nd ed. (IEEE, New York, 1991), Chap. 4, p. 247.

${ }^{17}$ J. R. Suckling, A. P. Hibbins, M. J. Lockyear, T. W. Preist, J. R. Sambles, and C. R. Lawrence, Phys. Rev. Lett. 92, 147401 (2004).

${ }^{18}$ Ansoft's High Frequency Structure Simulator, www.ansoft.com

${ }^{19}$ A. P. Hibbins, M. J. Lockyear, and J. R. Sambles, J. Appl. Phys. 99, 124903 (2006).

${ }^{20}$ D. Sievenpiper, L. J. Zhang, R. F. J. Broas, N. G. Alesopolous, and E. Yablonovitch, IEEE Trans. Microwave Theory Tech. 47, 2059 (1999). 